\title{
SARS-CoV-2 and Saliva as a Diagnostic Tool: A Real Possibility
}

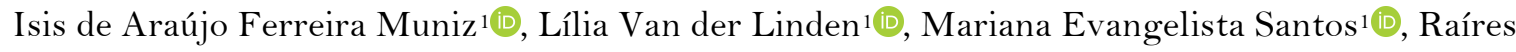

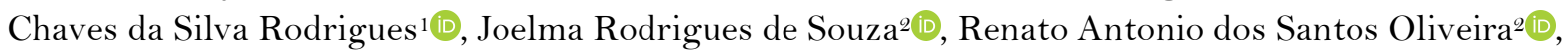
Lucio Roberto Cançado Castellano ${ }^{1}[0$, Paulo Rogério Ferreti Bonan 1 (10

${ }^{1}$ Post-Graduate Program in Dentistry, Federal University of Paraíba, João Pessoa, PB, Brazil.

${ }^{2}$ Department of Physiology and Pathology, Federal University of Paraíba, João Pessoa, PB, Brazil.

Author to whom correspondence should be addressed: Paulo Rogério Ferreti Bonan, Clinical and Social Dentistry, Federal University of Paraíba, Castelo Branco, João Pessoa, Paraíba, Brazil. 58051-900. Phone: +55 8332167250. E-mail: pbonan@yahoo.com.

Academic Editor: Yuri Wanderley Cavalcanti

Received: 02 June 2020 / Accepted: 24 June 2020 / Published: 06 July 2020

How to cite this article: Muniz IAF, Linden LV, ME, Rodrigues RCS, Souza JR, Oliveira RAS, et al. SARS-CoV-2 and saliva as a diagnostic tool: a real possibility. Pesqui Bras Odontopediatria Clín Integr. 2020; 20(supp1):e0112. https://doi.org/10.1590/pboci.2020.126

\begin{abstract}
Covid-19 is a respiratory disease caused by the SARS-CoV-2 virus. The high rate of contagion and the spread of the virus in the population make the early detection of the pathogen the means for the adequate targeting of infection control measures. WHO directs sample collection on upper respiratory specimens, including nasopharyngeal and oropharyngeal swab or wash in ambulatory patients, as well as lower respiratory specimens: sputum and/or endotracheal aspirate or bronchoalveolar lavage, in addition to citing blood and feces. Among the various sample collection methods, saliva has been investigated and reported as a potential source for diagnosis. Thus, we propose to evaluate the current scenario, based on recent publications on the perspective of detecting SARS-CoV-2 in saliva as a diagnostic method for Covid-19. The detection of SARS-CoV-2 through saliva seems to be very promising, although obstacles such as the technique and the location of the collection and the sample size of the research carried out so far may present a limitation for its use. The current scenario presents saliva as a reliable method for the detection of SARS-CoV-2, due to the ease of obtaining the samples, the possibility of self-collection, low cost because there is no need to use specific equipment, in addition to reducing the risk of transmission for health professionals.
\end{abstract}

Keywords: Saliva; Diagnosis; SARS Virus; Coronavirus Infections. 


\section{Introduction}

The coronavirus disease 2019 (Covid-19) is a respiratory disease considered a pandemic by the World Health Organization (WHO), caused by the severe acute respiratory syndrome-related coronavirus 2 (SARSCoV-2), so designated by the International Committee of Taxonomy of Viruses (ICTV), and initially named 2019 novel coronavirus (2019-nCoV). This new pathogen, characterized as an enveloped virus with a singlestranded RNA genome, belongs to the realm Riboviria, order Nidovirales, suborder Cornidovirineae, family Coronaviridae, genus Betacoronavirus and to the species of severe acute respiratory syndrome-related coronavirus [1,2]. Among the four existing genera of the coronavirus, Alpha- and Beta- are those that can infect mammals. It starts binding to host cells, and through the viral RNA replication process, it produces virions and releases by exocytosis for new infections. For this, structural proteins are needed for virion assembly, and the spike glycoprotein present on the virion surface is responsible for its binding to the host receptor. Thus, susceptibility to contagion, as well as the disease progression, can also be influenced by aspects of the host itself [3].

According to WHO, laboratory tests in suspected cases should be performed using samples collected from the upper respiratory tract, such as nasopharyngeal and oropharyngeal swab or wash in ambulatory patients, as well as lower respiratory specimens: sputum and/or endotracheal aspirate or bronchoalveolar lavage in patients with severe respiratory disease, in addition to blood, feces, and urine [4]. However, these diagnostic methods have some disadvantages, such as patient discomfort and bleeding or coughing, may occasionally occur, risk of contamination by healthcare professionals, the need for specific equipment, in addition to the limitation of sputum samples, since they are rarely present $[1,5]$.

An alternative to these collection methods that have been discussed in the studies is the saliva [1,6,7]. The approach of this biofluid as a potential alternative to replace the others, such as nasopharyngeal swabs or blood samples, is based on the possibility of its use for the extraction of viral RNA, exposing its viability in the diagnosis and mass detection of SARS-CoV-2 [1]. Thus, the variety of components present in saliva, including virus and antibodies, plus the possibility of freezing and storing these samples without compromising quality for years, could allow the monitoring of infection and the identification of the population's immunity to the virus, and with this reduces the spread of infection by asymptomatic individuals [7]. Some authors bring RTqPCR as the method of choice in the identification of the virus in respiratory secretions and blood, but due to a false negative that cannot be neglected, they propose an association with computed tomography, despite the limitations, in addition to complementary methods such as detection by antibodies [6].

The saliva has been reported as a non-invasive method, with significant advantages, such as lower cost, easy-to-obtain sample, the possibility of self-collection, lower risk of contamination. In addition, there is no need for trained staff for sample collection $[1,6,7]$. The high rate of contagion and the spread of the virus in the population make the early pathogen detection he most effective mean for the adequate targeting of infection control measures. Among the various sample collection methods, saliva has been reported as a potential source for diagnosis. Thus, we propose to evaluate the current scenario, based on recent publications on the perspective of detecting SARS-CoV-2 in saliva as a diagnostic method for Covid-19.

\section{Detection of Respiratory Viruses in Salivary Samples Before Covid-19}

Nasopharyngeal samples are the most effective samples for identify viruses that cause respiratory tract infection, being used both for diagnosis and in research as a comparative method with saliva [8,9]. 
Previously to the Covid-19 pandemic, saliva was already the focus of research to use it for diagnostic purposes in respiratory infections. In 2004, faced with a lack of knowledge about SARS-associated coronavirus (SARS-CoV) in oral droplets, Wang et al. examined samples of throat wash and saliva from patients considered suspect, using the quantitative real-time reverse transcription-polymerase chain reaction (RT-PCR) assay, and verified the presence of SARS-CoV RNA in all available specimens, and saliva presented the highest amount of virus RNA [10]. Also, the immunofluorescence assay was able to detect the pathogen in epithelial cells from the throat wash. This could lead to disseminating the oropharynx and oral cavity after viral replication. Thus, despite the limitations of the study concerning the sample size, they supported the indication of these samples as a diagnostic method for SARS-CoV-2, also because they were able to detect the presence of the virus in early samples even before the appearance of lung lesions, suggesting transmission by oral droplets by asymptomatic patients [10].

Previous studies have demonstrated the effectiveness of saliva in identifying these viral agents in the face of respiratory virus infections, with a high agreement with nasopharyngeal samples [8,9,10]. This method of collecting respiratory viruses was evaluated in two cohorts to observe the distinction between the viral loads of nasopharyngeal and saliva specimens. The detection rate for the first result was $91.8 \%$ and the second $57.1 \%$ for saliva. However, the reevaluation of patients who tested negative for the nasopharynx obtained a positive result for nine patients' saliva without identification in the nasopharynx, which could significantly affect the infection control measures. Thus, saliva should be considered as a diagnostic method [8].

The diagnostic validity of saliva demonstrated a $93 \%$ equivalence between the results in the identification of respiratory viruses, influenza, and respiratory syncytial virus, between saliva and nasopharyngeal aspirate, with $90.8 \%$ sensitivity and $100 \%$ specificity for saliva [9]. Saliva data on time and cost were also assessed and were 2.26 times faster and 2.59 times cheaper. And so, a possible way to be performed not only in diagnosis, but also in research [9].

\section{SARS-CoV Infection via Saliva}

The literature exposes the affinity of SARS-CoV-2 to the angiotensin-converting enzyme 2 (ACE2) receptor present in the host cell membrane, which can bind through a layer of proteins that surrounds it, which helps this connection facilitating their entry into human cells [3,11]. Thus, the expression of this receptor in cells can drive the possible pathways for the virus to enter the human body. The expression of ACE2 in the epithelial cells of the salivary gland has been confirmed and this makes them liable to be infected by SARSCoV-2, even though they are present in a smaller amount compared to other tissues, such as gastrointestinal system and renal and cardiac muscles. This result allows the identification of the virus in saliva, with a potential risk of transmission [3].

The analysis of public bulk-seq RNA datasets, in different tissues that express ACE2, showed that ACE2 is highly expressed in the oral mucosa, with the greatest expression in the oral tongue, floor of mouth, base of tongue, and among cells, epithelial cells, T cells, and fibroblasts. Moreover, minor salivary glands express higher levels of ACE2 than that in the lungs, suggesting that the oral cavity is very susceptible to infection acting as a potential target for the virus, and the salivary gland could be a major source of the virus in saliva. The identification of SARS-CoV-2 contamination pathways in the human body helps to understand the pathogenesis of the disease and provides support for prevention. However, the absence of a histological assessment is a limitation for these assessments [12,13]. Transmission occurs between humans normally 
through infected respiratory droplets, aerosols, sneezing or coughing, in direct contact with contaminated surfaces with the mouth, nose, or conjunctiva of the eyes [11].

In this sense, saliva presents a risk of transmission to dentists and other health professionals, since salivary samples may contain the virus in the process of replication, possibly its contagion configuration. Thereby, there is the possibility of being a diagnostic means for early detection of SARS-CoV-2, and it has also become a path for the use of proteomics, metabolomics, and antibody detection $[14,15]$.

\section{Current Scenario for the Identification of SARS-CoV-2 in Saliva}

With the emergence of SARS-CoV-2, because of the simplicity of acquiring the samples added to all the advantages concerning the reduction of the possibility of contagion of health professionals, already described and supported in previous studies, the diagnosis through saliva made even more relevant in the current scenario and new studies have emerged to evaluate this method. Previous authors detected SARS-CoV2 in $91.7 \%$, corresponding to 11 out of 12 patients evaluated, and argued in their study that saliva is a means of transmission in the positive test case for viral culture, since it can be expelled through droplets, in cough or even in cases with no respiratory symptoms. This could indicate the possibility of infection of the salivary gland, although they emphasize the diversity of saliva composition, which also consists of secretions from the nasopharynx or lung [5].

However, a discussion about the probability of identifying SARS-CoV-2 in saliva stated that the interpretation of the results of the study mentioned above could not be extrapolated through the statement that this method would result in the identification of the virus in $90 \%$ of infected patients, but detection of approximately $60 \%$ to $99 \%$, due to the small number of individuals tested. However, they recognize the relevance of the data and the need to continue studies with a larger sample size so that it is possible to resolve the questions about the sensitivity of this diagnostic method [16].

The diagnostic method through saliva is exposed in a very advantageous way, and research is constant to confirm the detection of the virus in saliva. With that, it is possible to observe that there must be a comparison of the results found in the tests with the diagnoses established by laboratories and confirmed through already established collections, such as nasopharyngeal rRT-PCR swabs. The Polymerase Chain Reaction with real-time reverse transcription (rRT-PCR) is the main test in identifying SARS-CoV-2 [17].

Previous authors performed collection through the drooling technique of 25 patients, due to the possibility of collecting only oral fluids instead of mucous secretions from the oropharynx or lower respiratory tract. Two hypotheses arose regarding the identification of the virus in saliva, the possible migration of the virus from the nasopharynx or the respiratory tract, or through the secretion of the salivary glands. They also affirmed the reliability of saliva in detecting SARS-CoV-2 and the possibility of using it in a rapid test, in addition to acquiring viral load in asymptomatic patients, to verify a threshold in the sensitivity of the test $[17]$.

In a recent study evaluating the SARS-CoV-2 viral load of 23 patients with Covid-19, using selfcollected posterior oropharyngeal saliva samples, $87 \%$ had the virus detectable in their saliva, in addition to reporting this viral load in the first week after the onset of symptoms and subsequent decline over time, as well as checking the presence of viral RNA in these same samples for more than 20 days in 7 patients. This implies that this type of sample allows its use both for the initial diagnosis and for monitoring the SARS-CoV-2 viral load. A possible relation was also observed between the high viral load detected after the onset of symptoms and the transmission of the disease during this period, regardless of the severity of the symptoms [18]. 
Differently, Chen et al. associated the presence of the virus in saliva with the severe form of the disease, in which, through the collection directly from the salivary glands as a way to obtain exclusively saliva samples, obtained a positive result of $12.90 \%$ of the patients, 3 of whom were very weak, indicating greater severity of the disease in cases of the presence of SARS-CoV-2 in the saliva from the salivary glands [3].

The possibility of releasing the virus after remission of symptoms makes it important to monitor the viral load, as well as the profile of antibodies, as they may not be directly proportional, and this may be relevant in cases not detectable by RT-PCR due to low viral load, in which the serological diagnosis would indicate the serum amount of IgG or IgM antibodies [18]. Zhang et al. evaluated oral swabs in the detection of the virus during the onset of the disease and did not consider it as a diagnostic method since negative results do not exclude the presence of the virus, and with that, serological tests, $\operatorname{IgG}$ and $\operatorname{IgM}$ would be indicated to confirm the infection [19].

Recently, an evaluation of 39 patients positive for PCR, 33 were identified with SARC-CoV-2 in saliva, affirming the viability of saliva as a non-invasive diagnostic method, useful for carrying out screening, due to the low cost, and the lack of adequate individual protective equipment for the collection of nasopharyngeal aspirates, restricting them to highly suspect patients [20].

In general, saliva is collected in a sterile container, and $2 \mathrm{~mL}$ of viral transport medium (VTM) is added to the container and the volume of saliva used varies between 0.5 and $1 \mathrm{~mL}$ [8], or specimens can be resuspended in $2 \mathrm{~mL}$ of phosphate-buffered saline (PBS) and subsequently subjected to nucleic acid extraction [17]. The method of collecting saliva is quite different among the studies evaluated. Although all studies evaluate saliva samples, the variations in the collection technique between them make them quite different in terms of the data found. The techniques of cough out saliva from throat, expectorated saliva, drooling technique or use of a pipette, saliva collected directly from the salivary gland, as well as the place where the samples were collected, from the deep throat, oral cavity or salivary glands $[3,5,17]$.

These differences in the collection differ in the composition of the evaluated saliva, and consequently, in the detection of the virus in the sample. Depending on the technique used, there may be a mixture of saliva and secretions from the nasopharynx and the lower respiratory tract and thus interfere in the interpretation of the results, since it is not possible to distinguish whether the virus comes from the salivary glands, nasopharynx or tract lower respiratory system. Some studies bring this information as a limitation [18] or use the technique for the acquisition of saliva only [3], others cite it as an advantage since regardless of the origin or composition, it can increase the detection of the virus without promoting discomfort to the patient [5]. In addition to the studies' limitations, the low concentration of analytes compared to other fluids such as blood and the viral load present in patients less than other aspirates constitute limitations to tests through the collection of saliva $[1,8]$.

Regardless of the collection method, all the studies evaluated emphasized the benefits of this diagnosis, which can be performed without specialized collection equipment. According to the studies' results, and evaluating the possibility of detecting the virus in these different samples, the suggestion is that the saliva from the deep throat presents a greater possibility of identifying the virus and, consequently, capable of diagnosing the disease early [13]. Moreover, a study comparing SARS-CoV-2 detection in nasopharyngeal and saliva samples from confirmed COVID-19 patients demonstrated that the sensitivity of SARS-CoV-2 detection from saliva would be comparable to nasopharyngeal swabs in early hospitalization and would be more consistent during extended period of hospitalization [21]. 
There is unanimity in the possibility of detecting SARS-CoV-2 in saliva, although there is no parameter for the collection technique in the researches. So far, the detection of SARS-CoV-2 through saliva seems to be very promising, although obstacles such as the technique and the location of the collection and the sample size of the research carried out so far may present a limitation for its use. As a diagnostic method, and with all the advantages presented, saliva as an isolated diagnostic method is not yet fully supported and must be associated with other methods for confirmation.

The current scenario presents saliva as a reliable method for the detection of SARS-CoV-2, due to the ease of obtaining the samples, the possibility of self-collection, low cost because there is no need to use specific equipment, in addition to reducing the risk of transmission for health professionals $[1,5]$.

\section{Conclusion}

The studies that evaluated saliva showed good agreement with the gold standard used, aspirated nasopharyngeal, with the potential to replace other biological samples used. However, this sample collection tool has not been recommended by the WHO to date.

\section{Authors' Contributions}

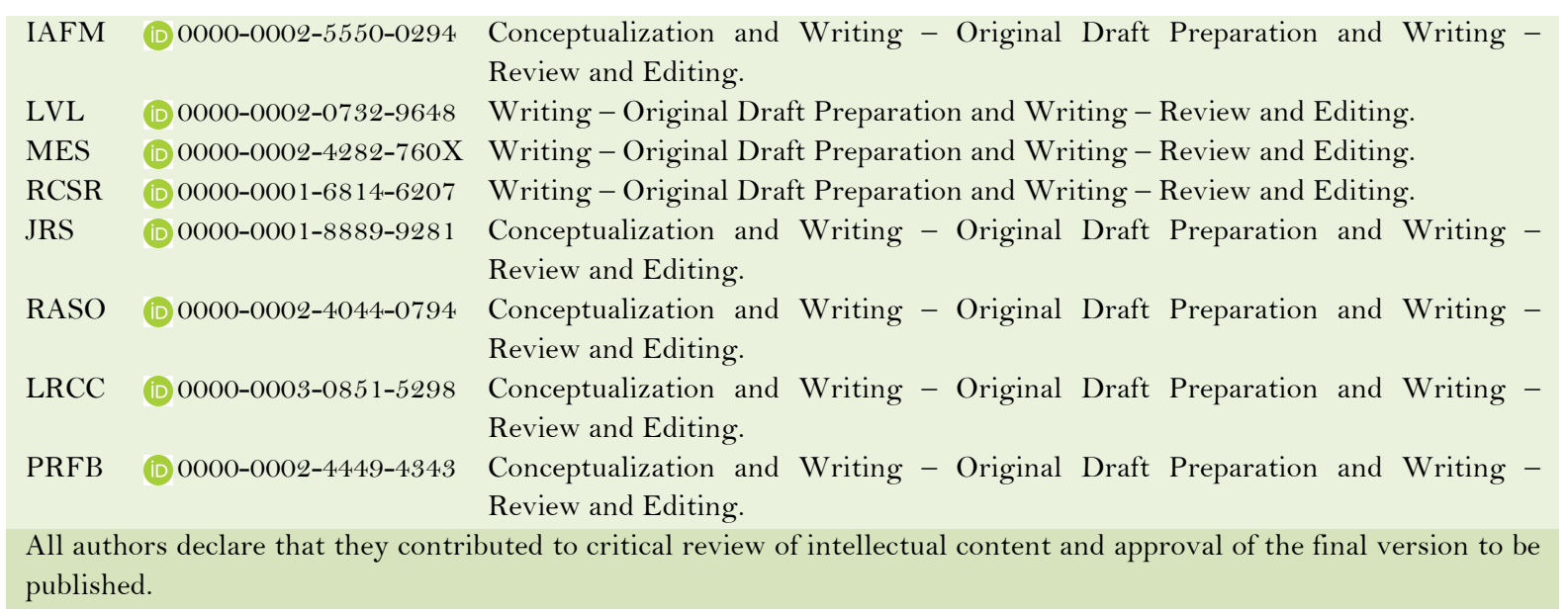

\section{Financial Support}

None.

\section{Conflict of Interest}

The authors declare no conflicts of interest.

\section{References}

[1] Sri Santosh T, Parmar R, Anand H, Srikanth K, Saritha M. A review of salivary diagnostics and its potential implication in detection of Covid-19. Cureus 2020; 12(4):e7708. https://doi.org/10.7759/cureus.7708

[2] Gorbalenya AE, Baker SC, Baric RS, Groot RJ, Drosten C, Gulyaeva AA, et al. Severe acute respiratory syndromerelated coronavirus: The species and its viruses - a statement of the Coronavirus Study Group. BioRxiv 2020. https://doi.org/10.1101/2020.02.07.937862

[3] Chen L, Zhao J, Peng J, Li X, Deng X, Geng Z, et al. Detection of 2019-nCoV in saliva and characterization of oral symptoms in COVID-19 patients. SSRN 2020. https://doi.org/10.2139/ssrn.3557140

[4] World Health Organization. Laboratory testing for coronavirus disease 2019 (COVID-19) in suspected human cases: interim guidance, 2 March 2020. Available from: https://apps.who.int/iris/handle/10665/331329. [Accessed on May 22, 2020]. 
[5] To KKW, Tsang OT, Yip CC, Chan KH, Wu TC, Chan JMC, et al. Consistent detection of 2019 novel coronavirus in saliva. Clin Infect Dis 2020; ciaa149. https://doi.org/10.1093/cid/ciaa149

[6] Li X, Geng M, Peng Y, Meng L, Lu S. Molecular immune pathogenesis and diagnosis of COVID-19. J Pharm Anal 2020. https://doi.org/10.1016/j.jpha.2020.03.001

[7] Han P, Ivanovski S. Saliva-friend and foe in the COVID-19 outbreak. Diagnostics 2020; $10(5): 290$. https://doi.org/10.3390/diagnostics 10050290

[8] To KKW, Lu L, Yip CCY, Poon RWS, Fung AMY, Cheng A, et al. Additional molecular testing of saliva specimens improves the detection of respiratory viruses. Emerg Microbes Infect 2017; 6(6):e49. https://doi.org/10.1038/emi.2017.35

[9] To KKW, Yip CCY, Lai CYW, Wong CKH, Ho DTY, Pang PKP, et al. Saliva as a diagnostic specimen for testing respiratory virus by a point-of-care molecular assay: a diagnostic validity study. Clin Microbiol Infect 2018; 1-7. https://doi.org/10.1016/j.cmi.2018.06.009

[10] Wang WK, Chen SY, Liu IJ, Chen YC, Chen HL, Yang CF, et al. Detection of SARS-associated Coronavirus in throat wash and saliva in early diagnosis. Emerg Infect Dis 2004; 10(7):1213-9. https://doi.org/10.3201/eid 1007.031113

[11] Odeh ND, Babkair H, Abu-Hammad S, Borzangy S, Abu-Hammad A, Abu-Hammad O. COVID-19: present and future challenges for dental practice. Int $J$ Environ Res Public Health 2020; 17:3151. https://doi.org/10.3390/ijerph 17093151

[12] Xu H, Zhong L, Deng J, Peng J, Dan H, Zeng X, et al. High expression of ACE2 receptor of 2019-nCoV on the epithelial cells of oral mucosa. Int J Oral Sci 2020; 12(1):8. https://doi.org/10.1038/s41368-020-0074-x

[13] Xu R, Cui B, Duan X, Zhang P, Zhou X and Yuan Q. Saliva: potential diagnostic value and transmission of 2019nCoV. Int J Oral Sci 2020; 12:11. https://doi.org/10.1038/s41368-020-0080-Z

[14] Braz-Silva PH, Pallos D, Giannecchini S, To KKW. SARS-CoV-2: What can saliva tell us? Oral Dis 2020. https://doi.org/10.1111/odi.13365.

[15] Sabino-Silva R, Jardim ACG, Siqueira WL. Coronavirus COVID-19 impacts to dentistry and potential salivary diagnosis. Clin Oral Invest 2020; 24:1619-21. https://doi.org/10.1007/s00784-020-03248-X

[16] Thompson RN and Cunniffe NJ. The probability of detection of SARS-CoV-2 in saliva. Stat Methods Med Res 2020; 29(4):1049-50. https://doi.org/10.1177/0962280220915049

[17] Azzi L, Carcano G, Gianfagna F, Grossi P, Gasperina DD, Genoni A, et al. Saliva is a reliable tool to detect SARSCoV-2. J Infect 2020; 81(1):e45-e50. https://doi.org/10.1016/j.jinf.2020.04.005

[18] To KKW, Tsang OTY, Leung WS, Tam AR, Wu TC, Lung DC, et al. Temporal profiles of viral load in posterior oropharyngeal saliva samples and serum antibody responses during infection by SARS-CoV-2: an observational cohort study. Lancet Infect Dis 2020; 20(5):565-74. https://doi.org/10.1016/S1473-3099(20)30196-1

[19] Zhang W, Du RH, Li B, Zheng XS, Yang XL, Hu B, et al. Molecular and serological investigation of 2019-nCoV infected patients: implication of multiple shedding routes. Emerg Microbes Infect 2020; 9(1):386-9. https://doi.org/10.1080/22221751.2020.1729071

[20] Williams E, Bond K, Zhang B, Putland M, Williamson DA. Saliva as a non-invasive specimen for detection of SARSCoV-2. J Clin Microbiol 2020. https://doi.org/10.1128/JCM.00776-20

[21] Wyllie AL, Fournier J, Casanovas-Massana A, Campbell M, Tokuyama M. Saliva is more sensitive for SARS-CoV-2 detection in COVID-19 patients than nasopharyngeal swabs. MedRXiV 2020. https://doi.org/10.1101/2020.04.16.20067835 